\title{
Memory as discrimination: What distraction reveals
}

\author{
C. Philip Beaman - Maciej Hanczakowski • Helen M. Hodgetts • \\ John E. Marsh • Dylan M. Jones
}

Published online: 10 May 2013

(C) Psychonomic Society, Inc. 2013

\begin{abstract}
Recalling information involves the process of discriminating between relevant and irrelevant information stored in memory. Not infrequently, the relevant information needs to be selected from among a series of related possibilities. This is likely to be particularly problematic when the irrelevant possibilities not only are temporally or contextually appropriate, but also overlap semantically with the target or targets. Here, we investigate the extent to which purely perceptual features that discriminate between irrelevant and target material can be used to overcome the negative impact of contextual and semantic relatedness. Adopting a distraction paradigm, it is demonstrated that when distractors are interleaved with targets presented either visually (Experiment 1) or auditorily (Experiment 2), a within-modality semantic distraction effect occurs; semantically related distractors impact upon recall more than do unrelated distractors. In the semantically related condition, the number of intrusions in recall is reduced, while the number of correctly recalled targets is simultaneously increased by the presence of perceptual cues to relevance (color features in Experiment 1 or speaker's gender in Experiment 2). However, as is demonstrated in Experiment 3 , even presenting semantically related distractors in a language and a sensory modality (spoken Welsh) distinct from that of the targets (visual English) is insufficient to eliminate false recalls completely or to restore correct recall to levels seen with unrelated distractors . Together, the study shows
\end{abstract}

C. P. Beaman $(\square)$

School of Psychology \& Clinical Language Sciences,

University of Reading, Earley Gate, Whiteknights,

Reading, RG6 6AL, UK

e-mail: c.p.beaman@reading.ac.uk

M. Hanczakowski • H. M. Hodgetts · J. E. Marsh • D. M. Jones

School of Psychology, Cardiff University, Cardiff, UK

J. E. Marsh

School of Psychology, University of Central Lancashire,

Preston, UK how semantic and nonsemantic discriminability shape patterns of both erroneous and correct recall.

Keywords Memory · Discrimination · Distraction . Front-end control $\cdot$ Retrieval orientation

Errors of memory can take a variety of forms. One common type of a memory error is simply unsuccessful retrieval of information from memory. For example, when a person is struggling to remember where his or her car is parked, a lack of information in his or her mind concerning the whereabouts of the car constitutes a clear failure of memory retrieval. Another broad class of memory failures consists of erroneous retrieval of contextually inappropriate information. The person can have a clear image of parking on a spot under a big oak. But does it necessarily mean that his or her car is now parked there? Or maybe he or she was planning to park there - the mental image of this plan serving as the basis for remembering - but because there was no space, he or she parked somewhere else. Or perhaps the car was parked there yesterday, an event that he or she now remembers, and today he or she parked closer to her workplace. As these examples show, accurate memory is not only about retrieval of relevant information, but also about not retrieving information that, despite being similar to sought information, is contextually inappropriate.

Although the generation of inappropriate false memories has often been investigated independently of the process of accurate retrieval, recent theorizing has conceptualized retrieval as a process of discrimination between relevant and irrelevant information (Goh \& Lu, 2012; Hunt, 2003; Nairne, 2005; Poirier et al., 2012; although note that there is a long tradition of viewing memory in this way-e.g., buildup and release from proactive interference (PI) are straightforwardly interpreted as consequences of a gradual decrease and then a sudden increase in discriminability; Watkins \& Watkins, 1975; Wickens, Born, \& Allen, 1963). According to this formulation, conditions that improve memory discrimination 
should reduce the incidence both of erroneous retrieval and of failures to retrieve. Memory discrimination is facilitated when more diagnostic cues are available, which tap directly into sought information to the exclusion of irrelevant information (cf. Nairne, 2002). Thus, conditions that offer diagnostic cues discriminating between relevant and irrelevant information should lead to both reduced erroneous retrieval and enhanced correct retrieval. The present study adopts the memory-asdiscrimination framework and investigates the consequences of an interplay between semantic and nonsemantic discriminability of relevant and irrelevant information for both erroneous and correct retrieval.

A number of ostensibly different approaches (e.g., sourceconstrained retrieval, retrieval orientation, and diagnostic monitoring) are all related to this cue diagnosticity idea that retrieval of items is determined by the adoption of cues that match the target information and result in the exclusion of nontarget information. However, the specific implementation of some of these otherwise similar approaches can lead to differing predictions. For example, Jacoby and colleagues (Jacoby, Kelley, \& McElree, 1999; Jacoby, Shimizu, Daniels, \& Rhodes, 2005; Jacoby, Shimizu, Velanova, \& Rhodes, 2005) proposed a mechanism called sourceconstrained retrieval - the focusing of retrieval to restrict information that comes to mind to that which originates from the desired source. This focusing is accomplished by mentally reinstating retrieval cues from the source context that specifically fit the way in which the target information was encoded. This is a specific instance of a mechanism of front-end control, where front-end refers to the suggestion that rememberers enhance recall accuracy by preventing the retrieval of incorrect information. It can be contrasted with back-end control, which suggests that monitoring and control processes are used to edit out incorrect information only after it has been retrieved (Halamish, Goldsmith, \& Jacoby, 2012; Higham \& Tam, 2005; Jacoby et al. 2005a, b; Koriat \& Goldsmith, 1996; Shimizu \& Jacoby, 2005). Back-end control includes such proposals as Jacoby, Kelley, and Dywan's (1989) attributional framework, Whittlesea's SCAPE (production-evaluation) framework (Leboe \& Whittlesea, 2002; Whittlesea, 1997), and memory editing mechanisms such as the distinctiveness heuristic (Dodson \& Schacter, 2001, 2002; but see Gallo, Weiss, \& Schacter, 2004) and recollection rejection (Brainerd, Reyna, Wright, \& Mojardin, 2003).

Importantly, the back-end control theories do not postulate a necessary link between erroneous and correct retrieval. According to back-end control theories, editing out of erroneous information occurs at the postretrieval stage of deciding whether retrieved information meets the criteria of retrieval. Thus, any observed reduction in incidence of erroneous responses due to more efficient back-end control occurs subsequent to - and is independent of - the processes that lead to correct retrieval. Front-end and back-end control may coexist within a single model. For example, the feature model of immediate serial recall (Nairne, 1990) makes use of Luce's choice rule to select the most likely secondary memory target from the cues available within primary memory (front-end control), but, postretrieval, there is a bias against repeatedly recalling the same item, should that item appear to be the most likely target according to Luce's rule (a form of back-end control). In free-report situations, however, much work (e.g., Goldsmith \& Koriat, 2008) has emphasized the back-end role of postretrieval monitoring and control processes. When these mechanisms fail, the result is a commission error or false memory, but there is no necessary consequence for the incidence of correct recall, because all of these back-end mechanisms operate to edit out inappropriate responses after retrieval has occurred. Arguably, however, at least some of the blame for commission errors in free report should be assigned to the faulty retrieval processes that brought the incorrect information to mind in the first place. Commission errors of this type, however, are also likely to have consequences for the incidence of correct recall, because such frontend control processes occur prior to retrieval.

In the experiments reported here, we explore retrieval as a discrimination process using a novel method-namely, the study of semantic distraction during free recall-with the aim of determining whether perceptual cues to the source of the items can be employed effectively during a task that is otherwise dominated by semantic processing (in which case they should impact on erroneous recall) and whether these are employed as front-end (in which case they could also impact on correct retrieval) or back-end (in which case they should not) control. Before describing our study, however, we briefly discuss previous work on the relationship between correct and erroneous retrieval as related to memory-as-discrimination and front-end control frameworks.

Two studies, by Alban and Kelley (2012) and by Kantner and Lindsay (2013), are directly relevant to the issue of correct recall and front-end control. Alban and Kelley used a memory-for-foils procedure to implement conditions under which people were likely to enrich a retrieval cue with diagnostic details pertaining to the source of to-be-remembered items (front-end control). Importantly, when Alban and Kelley contrasted two conditions for which evidence from their memory-for-foils procedure indicated a difference in diagnosticity of the adopted retrieval cues, no difference in hit rates between these conditions was observed. These results thus fail to show a direct benefit for correct retrieval despite evidence for the adoption of seemingly diagnostic cues at retrieval. Similarly, Kantner and Lindsay looked directly at the issue of whether "source-constrained search" leads to selective recollection of details of target items at a recognition test, but the predicted effect was apparent in only one of four experiments. Even when participants tried to constrain their memory search to items from a certain source, recollection for 
the items from this source was no different to recollection for the items coming from the other, irrelevant source. Thus, this study also fails to provide evidence that more diagnostic cues aid correct retrieval. However, both of these studies used recognition tests that required re-presentation of probes. Since, in recall, probes from the inappropriate source are not presented at test - and thus cannot trigger the kind of automatic retrieval that may undermine the effectiveness of source-constrained search in recognition - there are more opportunities to find evidence of front-end control. By concentrating on recall, it may be possible to show what the studies by Alban and Kelley and by Kantner and Lindsay could not show with recognition, that constraining retrieval to a certain source with the help of distinctive information not only reduces false retrieval, but also increases correct retrieval, consistent with front-end, source-constrained retrieval.

Both erroneous recognition and erroneous recall (and, in turn, discrimination processes) are often investigated via the Deese-Roediger-McDermott (DRM) paradigm (Deese, 1959; Roediger \& McDermott, 1995). Here, participants are shown a list of associates (e.g., bed, rest, awake, etc.) of a single, not-presented item (in this case, sleep), referred to as a critical lure. Recognition or free recall tests for the list usually result in erroneous endorsement or retrieval of the critical lure. This intrusion can be explained by supposing that when participants attempt to retrieve studied words, they incorporate the semantic gist of a study list into a cue they use to access memory (e.g., Brainerd, Wright, Reyna, $\&$ Mojardin, 2001). A critical lure is a good, valid match to such semantic elements of the cue and becomes erroneously incorporated into the recalled list. The DRM paradigm reveals the prominent role played by semantic processing: If individuals were not inclined to cue memory with semantically rich cues, false memory errors would not occur. Enriching studied items with perceptual details - for example, by providing pictures at study (Israel \& Schacter, 1997) - reduces the instances of erroneous retrieval. Presumably, nonsemantic information can be incorporated into a cue alongside semantic information, which leads to improved discrimination of relevant from irrelevant information even in the face of their high semantic similarity. However, these studies are often presented in the context of a "distinctiveness heuristic" (Schacter, Israel, \& Racine, 1999), a back-end control mechanism, which assigns reduction of false remembering to a strategy of examining, postretrieval, those details that differentiate between correct and incorrect information (but see Budson et al., 2005, for a front-end conceptualization of the heuristic).

The emphasis placed within the DRM paradigm on erroneous retrieval as the primary variable of interest makes it difficult to directly address the issue of primary interest here, the locus of discrimination control (front- or backend) when selecting a target or targets from among semantically related lures. From the standpoint of frontend control, erroneous and correct retrieval represent two facets of the same process; factors that increase the effectiveness of front-end discrimination, like nonsemantic discriminability of relevant and irrelevant information, should lead both to fewer instances of erroneous retrieval and to improved correct retrieval. Numerous studies that examined the role of perceptual discriminability in the DRM paradigm, using both recognition and recall, failed to document the enhancement of correct retrieval when erroneous retrieval was reduced, a result ostensibly inconsistent with the prediction of the front-end control framework (e.g., Hanczakowski \& Mazzoni, 2011; McCabe \& Smith, 2006; Schacter et al., 1999). However, in the DRM procedure, participants are required to discriminate a series of relevant information from a single, critical lure rather than from a similar series of semantically related but contextually irrelevant information. ${ }^{1}$ Thus, positive evidence of improved correct retrieval in the DRM paradigm may be elusive and is not necessarily anticipated, even with front-end control, when target information needs to be discriminated only from a single critical lure.

Instead, the present study employs a semantic distraction (SD) paradigm (Neely \& LeCompte, 1999) to investigate the role of semantic and nonsemantic discriminability in producing erroneous and correct retrieval. In this paradigm, participants study and subsequently recall categorized words that are accompanied by distractors (commonly presented in the auditory channel) that participants are asked to ignore. In the related condition, the distractors are words taken from the same category to which studied items belong, and in the unrelated condition, the distractors are words taken from a different category. The SD paradigm creates conditions under which the efficacy of retrieval, understood as the process of discrimination, becomes affected by strong semantic similarity of relevant and irrelevant information. Results from both DRM and SD paradigms attest to the effect of semantic similarity on erroneous retrieval. The high rate of intrusions of critical lures in the DRM paradigm is echoed by elevated levels of distractor intrusions in the related, as compared with the unrelated, condition of the SD paradigm (e.g., Beaman, 2004; Marsh, Hughes, \& Jones, 2008). Nonetheless, correct retrieval lies in the very center of interest for studies employing the SD paradigm, where presentation of related distractors increases intrusions and, at the same time, decreases the rate of correct recall, as compared with presentation of unrelated distractors (e.g., Marsh, Beaman, Hughes, \& Jones, 2012; Marsh, Hughes, \& Jones, 2008, 2009; Marsh, Sörqvist, Beaman, \& Jones, in

\footnotetext{
${ }^{1}$ We assume that a feature common to a whole class of items may serve the purpose of differentiating between what is relevant and what is irrelevant.
} 
press; Neely \& LeCompte, 1999). Thus, in line with the memory-as-discrimination framework, when at retrieval relevant and irrelevant information share strong semantic similarity, participants find it difficult not only to avoid retrieval of irrelevant information but also to retrieve relevant information. This implies a mechanism of front-end control responsible for discriminating between the relevant material and the related distractors.

In the SD paradigm, the data strongly suggest that the prevailing cues for recall are semantic (because a semantic distraction effect occurs) and temporal-contextual (because it is limited in scope to those distractors occurring within a single trial). If participants are able to utilize extra cues that are not semantic-contextual, these could, in principle, also enable such exclusion and so should result in an analogous reduction in intrusions in the related condition. More intriguing is whether increasing nonsemantic discriminability additionally results in reduced incidents of failed retrieval of correct information. The discrimination perspective on memory retrieval as a front-end mechanism clearly suggests so, whereas back-end control makes no such prediction.

The present study includes three experiments employing the SD paradigm and designed to assess the role of nonsemantic discriminability on correct and erroneous retrieval under conditions of high semantic similarity of relevant and irrelevant information. Experiments 1 and 2 provide tests of the hypothesis, derived from the discrimination framework of memory retrieval, that enhancing nonsemantic discriminability of semantically similar relevant and irrelevant information should lead to reduction in instances of erroneous retrieval (a decrease in false recall of irrelevant information consistent with either front-end or back-end control) and in failures of correct retrieval (an increase in correct recall of relevant information predicted only by front-end control). Experiment 1 tested this hypothesis in the visual modality, and Experiment 2 tested the same hypothesis in the auditory modality. Experiment 3 used two different dimensions to enhance nonsemantic discriminability of relevant and irrelevant information to assess whether errors caused by increased semantic similarity can be completely eliminated with the help of cuing with nonsemantic details.

\section{Experiment 1}

The present experiment employs the SD paradigm in the visual modality and, apart from manipulating semantic similarity of relevant and irrelevant information, it manipulates nonsemantic discriminability of this information. Participants studied to-be-remembered (TBR) words from a single category that were interleaved with to-be-ignored (TBI) words presented in a different type of font. In the related condition,
TBR and TBI words were taken from the same category, whereas in the unrelated condition, TBR and TBI words were taken from different categories. Critically, in the condition of high nonsemantic discriminability, TBR words were presented in a different color than TBI words, whereas in the condition of low nonsemantic discriminability, all words were presented in the same color. On the basis of the previous findings from the SD paradigm (e.g., Beaman, 2004; Marsh et al., 2008), we predicted that the process of memory discrimination would be impaired in the related, as compared with the unrelated, condition, leading to both more instances of erroneous retrieval and more instances of failures of correct retrieval in the related condition. This result would testify to the first general characteristic of memory discrimination, which is the sensitivity of this process to semantic similarity between relevant and irrelevant information.

Of most interest for the present study is, however, the second general characteristic, which is that in the face of increased semantic similarity, nonsemantic details that differentiate between relevant and irrelevant information can be used to aid the process of discrimination. We hypothesized that introducing such nonsemantic details, which in this case different were colors used for TBR and TBI words, should lead to enhanced discrimination, which should manifest itself with both a reduction in erroneous retrieval and an increase in correct retrieval obtained in the related condition.

\section{Method}

\section{Participants}

Forty-eight students at Cardiff University participated in the study as part of a course requirement; 24 were assigned to the related condition, and 24 to the unrelated condition. All were native English speakers and reported normal vision and hearing.

\section{Materials and design}

Stimuli were chosen from 37 semantic categories of the Van Overschelde, Rawson, and Dunlosky (2004) category norms. Items from positions $16-30$ were used as TBR words, and items from positions $1-15$ were used as TBI words. Each participant completed 18 trials of learning a list of words from a single category and performing an immediate recall test for these words. In the related condition, TBI words, which were presented in the spaces between presentations of TBR words, were taken from the same category as TBR words. Thus, in the related condition, 18 category lists were used. In the unrelated condition, TBI words were taken from a different category than were TBR words. Thus, in the unrelated condition, 18 category lists 
were used for study, and the remaining 18 categories were used as a source of TBI words. The counterbalancing was employed to rotate categories used as a source of TBR or TBI words.

For both groups of participants, 9 of the 18 trials were included in the low nonsemantic discriminability condition, and the remaining 9 trials were included in the high nonsemantic discriminability condition. For the low nonsemantic discriminability condition, all TBR and TBI words were presented in a blue font color, and for the high nonsemantic discriminability condition, all TBR words were presented in a blue font, whereas all TBI words were presented in a red font. The trials from both conditions were blocked, and the order of blocks was counterbalanced across participants.

The experiment yielded to a 2 (semantic similarity of TBR and TBI words: related vs. unrelated) $\times 2$ (nonsemantic discriminability of TBR and TBI words: high vs. low) mixed design with the first factor manipulated between participants and the second factor manipulated within participants.

\section{Procedure}

All participants were tested on individual computers. During each trial, TBR and TBI words were presented in the center of the screen in 72-point Times font. The TBR words were presented in normal font, while the TBI words were presented in italics. The words were presented at a rate of one per second. The color of the font was determined by the type of item and condition, as described earlier. Participants were instructed that regardless of font color, they were to remember the items written in normal font and to ignore the words in italics. After presentation of all items the computer screen displayed the word recall in green. Participants then had $30 \mathrm{~s}$ to write down as many of the TBR items as they could in any order on the response sheets provided. A tone signaled the end of the recall period, and participants were to press the space bar to initiate the next trial. At the start of the experiment, participants completed one practice trial and were given the opportunity to ask any questions before the onset of the experimental trials.

Results

\section{False recalls}

The predictions for the present set of analyses concerned mostly the level of intrusions of TBI words in the related condition, which we predicted would be reduced under conditions of high nonsemantic discriminability. The great majority $(85 \%)$ of intrusions in the related conditions were, in fact, intrusions of TBI words. Given the very small number of other intrusions, we decided not to analyze different types of intrusions separately but, rather, to analyze all types of intrusions jointly. ${ }^{2}$ Omission errors are not included because, in false recall, these are simply list length minus correct recall. The same approach was adopted in the other experiments.

The mean number of intrusions per participant ${ }^{3}$ was analyzed by a 2 (semantic similarity of TBR and TBI words: related vs. unrelated) $\times 2$ (nonsemantic discriminability of TBR and TBI words: high vs. low) ANOVA, which yielded significant main effects of semantic similarity, $F(1,46)=$ $12.18, M S E=32.19$, partial $\eta^{2}=.21, p=.001$, and of nonsemantic discriminability, $F(1,46)=6.56, M S E=$ 8.69, partial $\eta^{2}=.13, p=.014$. There was a significant interaction, $F(1,46)=10.14, M S E=8.69$, partial $\eta^{2}=.18$, $p=.003$ (see Fig. 1). Independent samples $t$-tests confirm that the effect of semantic relatedness was significant when nonsemantic discriminability was low, $t(46)=4.07, p<.001$, but not when it was high, $t(46)=1.89, p=.07$.

\section{Correct recalls}

The number of correctly recalled TBR words was analyzed with the same $2 \times 2$ ANOVA, which yielded a significant main effect of semantic similarity, $F(1,46)=9.93, M S E=104.07$, partial $\eta^{2}=.18, p=.003$, but no significant main effect of nonsemantic discriminability, $F(1,46)=3.62, M S E=18.19$, partial $\eta^{2}=.07, p=.06$. There was, however, a significant interaction, $F(1,46)=4.39, M S E=28.19$, partial $\eta^{2}=.09$, $p=.042$, which, as is shown by Fig. 2 , arose due to a greater difference in correct recall between related and unrelated conditions when nonsemantic discriminability was low, as compared with a condition in which it was high. In conditions of low discriminability, this difference was significant, $t(46)=$ $3.68, p=.001$, whereas for the high-discriminability condition, it was not, $t(46)=1.87, p=.07$. This result confirms the prediction that, in the face of high semantic similarity of TBR and TBI words, high nonsemantic discriminability leads to improved correct recall, as compared with low nonsemantic discriminability.

\section{Discussion}

The results of the first experiment are consistent with the predictions formulated on the basis of the memory-as-

\footnotetext{
${ }^{2}$ In fact, the results for all experiments remain unchanged if the analysis is limited to intrusions of TBI words. Intrusions of other kinds are too rare (zero intrusions in one or more conditions from 30/48 participants) for any independent analysis to be statistically meaningful.

${ }^{3}$ Proportions are not appropriate here because these are intrusions from a set of possible intrusion errors of unknown size. However, as an illustration of the rate of intrusion errors, across all conditions, intrusions expressed as a proportion of the number of TBI items experienced per participant ran at a rate of $13 \%$, on average.
} 


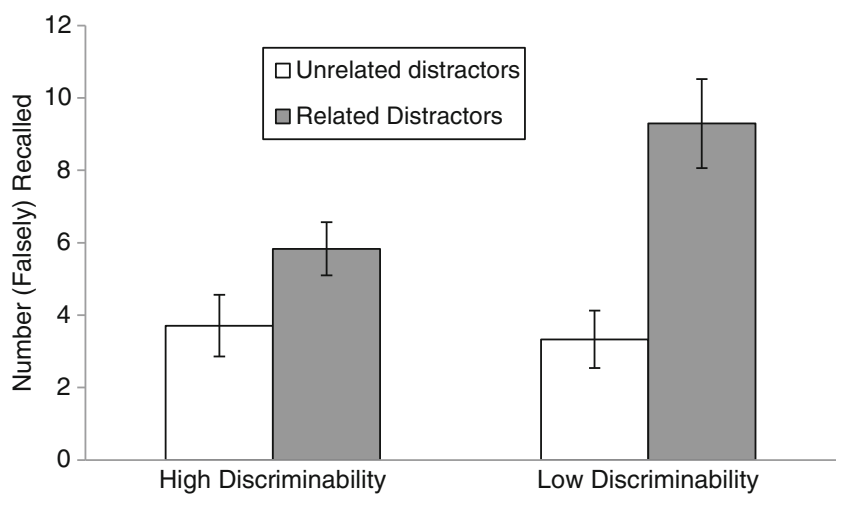

Fig. 1 The effects of semantic and perceptual relationships between TBR and TBI words on the number of intrusion errors (items falsely recalled) in Experiment 1. Error bars are standard errors

discrimination framework. First, the comparison of conditions where TBR and TBI items were either semantically related or unrelated clearly indicates that semantic similarity leads both to an increased number of intrusions and a reduced number of correct recalls. Second, the manipulation of nonsemantic discriminability demonstrated that when TBR and TBI items are semantically related, memory performance can be aided by associating TBR and TBI items with different variants of a nonsemantic feature. When TBR words were presented in a different color to TBI words, performance was enhanced, and, crucially for the present purpose, this enhancement was revealed by both reduction in intrusions and increase in correct recall. This pattern of results is consistent with the hypothesis that nonsemantic color details used here aided the process of discrimination between relevant (TBR) and irrelevant (TBI) information during memory retrieval that would otherwise be based upon semantic-contextual cues. These results are consistent with the results of the studies that have shown reduced erroneous retrieval of a single critical item when nonsemantic discriminability is enhanced (e.g., McCabe \& Smith, 2006; Schacter et al., 1999). Importantly, this basic finding is extended both by demonstrating the same effect when



Fig. 2 The effects of semantic and perceptual relationships between TBR and TBI words on the probability of correct recall in Experiment 1. Error bars are standard errors individual TBR items are discriminated from among a series of TBI distractors and by showing a simultaneous beneficial effect of increased nonsemantic discriminability for correct recall of the TBR items. The latter finding is predicted only by a frontend control hypothesis.

Although the results of Experiment 1 clearly support the predictions of the memory-as-discrimination framework, there is an alternative account of our findings. For nonsemantic discriminability, we argue that when a specific detail differentiates between relevant and irrelevant information, this detail becomes incorporated in the cue used to access memory at retrieval, which increases diagnosticity of the cue and enhances the discrimination between relevant and irrelevant information, resulting in reduced erroneous retrieval and increased correct retrieval. However, the effects of differentiating detail between TBR and TBI items could be attributed to encoding rather than retrieval mechanisms. For instance, the differentiating details may help participants to recruit different encoding strategies for TBR and TBI items. Because presentation of both types of items in Experiment 1 was visual, the simplest strategy participants could adopt would be to subtly divert their gaze or visual fixation whenever a TBI item was presented. It is possible that using different colors to mark TBR and TBI items could facilitate such a strategy, leading to impoverished encoding of TBI words. This, in turn, would render discrimination between competitors at retrieval easier, leading to the observed pattern of results. Experiment 2 was designed to test the retrieval and encoding hypotheses of the observed effects by implementing the SD paradigm in the auditory modality, which precludes simple avoidance strategies at encoding.

\section{Experiment 2}

Experiment 2 followed the design of Experiment 1, with a change in the modality of presentation of TBR and TBI words. Rather than visually, in Experiment 2, both TBR and TBI words were presented auditorily. TBR and TBI words were marked by the loudness of the presenting voice, with quiet words being marked as TBR and loud words being marked as TBI. As in Experiment 1, TBI words were either semantically related or unrelated to TBR words. The nonsemantic discriminability manipulation was introduced using speakers of different genders. For the low nonsemantic discriminability condition, both TBR and TBI words were spoken by the same female voice, whereas for high nonsemantic discriminability condition, TBR words were spoken by a female voice, and TBI words were spoken by a male voice. We predicted that the semantic similarity manipulation would lead to the same effects as in Experiment 1 and previous studies on semantic distraction (Beaman, 2004; Marsh et al., 2012; 
Marsh et al., 2008, 2009; Neely \& LeCompte, 1999) — namely, a higher level of intrusions and a lower level of correct recall for the related condition. Perhaps of greater interest is the effect of the nonsemantic discriminability manipulation. Because encoding of spoken distractors is obligatory (Hanley \& Bakopoulou, 2003; Salamé \& Baddeley, 1982) and cannot be avoided by divertive strategies, changing of the speaker's voice between conditions should not result in changes in the level of encoding of TBI words. Thus, if more effective avoidance strategies and associated changes in encoding of TBI words were solely responsible for the reduction in erroneous recall and the increase in correct recall observed in the high nonsemantic discriminability condition of Experiment 1, the effects of nonsemantic discriminability should not replicate here. Contrarily, if our initial argument is correct and the effects documented in Experiment 1 stemmed from more diagnostic cuing at retrieval (front-end control) in the high nonsemantic discriminability condition, we would predict that the same effects should emerge in the present experiment.

\section{Method}

\section{Participants}

Forty-eight students at Cardiff University took part in the study. Half were assigned to the unrelated condition, and half were assigned to the related condition. All reported normal or corrected-to-normal vision and normal hearing and spoke English as their first language. They received either course credit or payment for their participation.

\section{Materials, design, and procedure}

The same categorized lists of words as those used in Experiment 1 were used in the present experiment. Each word from these lists was recorded by a female voice, and additionally, all TBI words were recorded by a male voice. The experiment yielded again to a 2 (semantic similarity of TBR and TBI words: related vs. unrelated) $\times 2$ (nonsemantic discriminability of TBR and TBI words: high vs. low) mixed design with the first factor manipulated between participants and the second factor manipulated within participants.

The procedure followed the procedure of Experiment 1, except for the following changes. The presentation of all words was auditory. All TBR words were presented by a female voice at $60 \mathrm{~dB}(\mathrm{~A})$. TBI words were presented over headphones at $75 \mathrm{~dB}(\mathrm{~A})$, by a female voice in the low nonsemantic discriminability condition and by a male voice in the high nonsemantic discriminability condition.

\section{Results}

\section{False recalls}

A 2 (semantic similarity of TBR and TBI words: related vs. unrelated) $\times 2$ (nonsemantic discriminability of TBR and TBI words: high vs. low) ANOVA yielded a significant main effect of semantic similarity, $F(1,46)=20.48, M S E=40.16$, partial $\eta^{2}=.31, p<.001$, and also of nonsemantic discriminability, $F(1,46)=14.69, M S E=4.89$, partial $\eta^{2}=.24, p<.001$. There was also a significant interaction, $F(1,46)=5.12, M S E=4.89$, partial $\eta^{2}=.10, p=.028$ (see Fig. 3), although the semantic relatedness effect was significant for both conditions of low nonsemantic discriminability, $t(46)=4.70, p<.001$, and high nonsemantic discriminability, $t(46)=3.81, p=.001$.

\section{Correct recalls}

An equivalent ANOVA carried out on the items correctly recalled revealed a significant main effect of semantic similarity, $F(1,46)=47.00, M S E=157.13$, partial $\eta^{2}=.51, p<$ .001 , but no main effect of nonsemantic discriminability, $F(1,46)=2.46, M S E=29.9$, partial $\eta^{2}=.05, p=.12$. There was, however, a significant interaction, $F(1,46)=6.83$, $M S E=29.90$, partial $\eta^{2}=.13, p=.012$ (see Fig. 4), although independent $t$-tests indicate that the difference between semantically related and unrelated conditions remain statistically significant for both low nonsemantically discriminable, $t(46)=7.80, p<.001$, and highly discriminabl, $t(46)=4.96, p<.001$, conditions.

\section{Discussion}

The present experiment conceptually replicated Experiment 1 in a different modality and, thus, with a different feature differentiating between TBR and TBI words. Despite the change from visual to auditory modality, the results of the

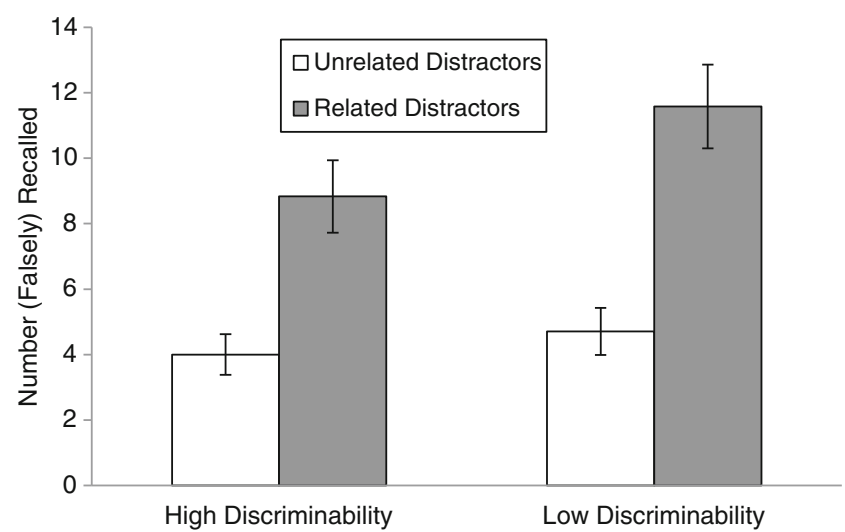

Fig. 3 The effects of semantic and perceptual relationships between TBR and TBI words on the number of intrusion errors (items falsely recalled) in Experiment 2. Error bars are standard errors 


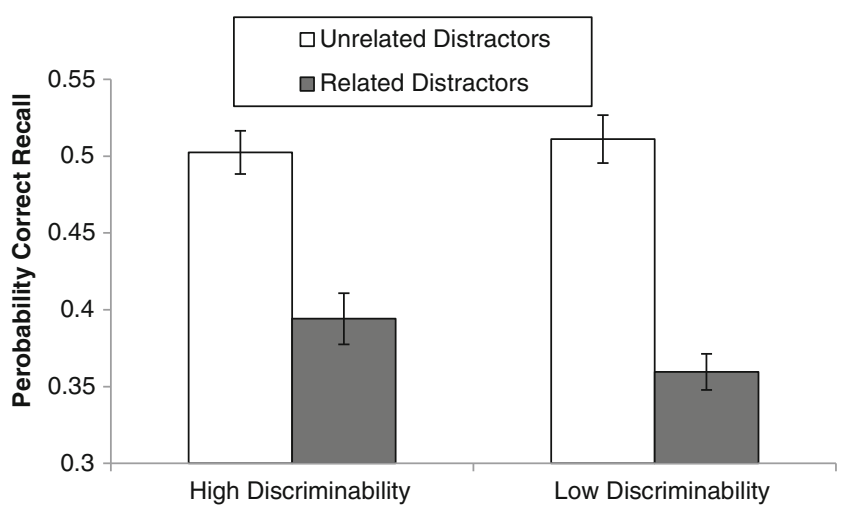

Fig. 4 The effects of semantic and perceptual relationships between TBR and TBI words on the probability of correct recall in Experiment 2. Error bars are standard errors

present experiment closely followed the results of Experiment 1. Semantic similarity of TBR and TBI words once again led to an increased number of intrusions and a simultaneous reduction in correct responding. Introducing a specific feature of gender of a speaker that differentiated between TBR and TBI words helped counteract the effects of increased semantic similarity. When TBR and TBI words were semantically related, presenting TBR and TBI words in a different voice led to a reduction in erroneous recall accompanied by an increase in correct responding, as compared with a situation in which the same voice was used to present both TBR and TBI words. Given that, in this experiment, participants could not simply avoid encoding auditorily presented TBI words, it is unlikely that presenting TBR and TBI words in different voices exerted its influence at encoding. The result of the present experiment are more consistent with our initial hypothesis that when a nonsemantic feature differentiates between semantically similar relevant and irrelevant information, this feature becomes included in the retrieval cue, which renders it more diagnostic and results in facilitated process of discrimination.

A notable feature of the results of both Experiments 1 and 2 is that although high nonsemantic discriminability helped to counter the effects of increased semantic similarity between TBR and TBI words in the related condition, it did not equate performance with the unrelated condition. In other words, although high nonsemantic discriminability reduced erroneous recall and increased correct recall in the related condition, participants in this condition still produced more intrusions and recalled fewer correct items, as compared with the unrelated condition in both Experiments 1 and 2. This pattern of results is similar to the pattern documented in the DRM paradigm. Although perceptually enriched studied items reduce false remembering of critical lures in that paradigm, such strongly semantically related critical lures are invariably erroneously remembered at the higher rate than foils that are semantically unrelated to studied items (e.g., Hanczakowski \& Mazzoni, 2011; Schacter et al., 1999). Additionally, however, Experiments 1 and 2 directly demonstrate that it is not simply perceptual enrichment of the TBR items but perceptual differentiation of the TBR from the TBI items that underlies this finding. The effect itself is also extended to cover discrimination of a series of TBR items from a series of semantically related TBI items by means of a single perceptual feature distinguishing the two series and is shown to affect the probability of correct, as well as erroneous, recall. The question that the next experiment addresses is whether, in the SD paradigm, the negative effects of semantic similarity between TBR and TBI items can be entirely eliminated when nonsemantic discriminability of TBR and TBI items is further enhanced.

\section{Experiment 3}

The present experiment sought to create conditions of maximal nonsemantic discriminability between TBR and TBI words in an attempt to completely negate the effects of semantic similarity on both erroneous and correct recall. If semantic information can be discarded completely as a cue, it should be possible to eliminate the SD effect completely. If, however, the semantic and conceptual encoding of categorical lists cannot be so discarded in favor of more diagnostic cues, then, even though semantic information may be actively misleading, it will be incorporated into the search set, and an SD effect will remain. To this end, two arguably distinct dimensions, rather than a single perceptual feature, were used to enhance nonsemantic discriminability of TBR and TBI words. First, to increase perceptual discriminability, TBR and TBI words were presented in different modalities. Both Experiments 1 and 2 presented TBR and TBI words in the same modality, varying only a relatively minor perceptual feature to increase nonsemantic discriminability. We reasoned that when TBR and TBI words are presented in different modalities, all types of perceptual features could contribute to nonsemantic discriminability of TBR and TBI words, which should provide much better conditions for counteracting semantic similarity of these words. However, from previous studies employing the SD paradigm, it is already clear that discrimination by modality per se is not sufficient to completely counteract semantic similarity. Previous studies using visual presentation of TBR items and auditory presentation of TBI items demonstrated small but consistent negative effects of semantic similarity between TBR and TBI words on memory performance (e.g., Marsh et al., 2008; Neely \& LeCompte, 1999). For this reason, in the present experiment, we introduced a second major dimension along which TBR and TBI words could be differentiated - that is, a linguistic dimension. In the present experiment, participants were bilinguals (Welsh-English), and 
the congruency of languages in which TBR and TBI words were presented was manipulated. Goggin and Wickens (1971) demonstrated a release from PI when the language within which to-be-recalled information was shifted, so if language of presentation could equally be used as a cue here, the SD effect might be reduced for these reasons. Of greatest interest was thus whether the negative effect of semantic similarity is eliminated when both modality and linguistic information differentiates between TBR and TBI ignored words and memory performance in the related condition is equated with performance in the unrelated condition.

Method

\section{Participants}

Twenty-eight Welsh-English bilinguals participated. They were students at Cardiff University who received course credit for their participation. All reported normal or corrected-to-normal vision and normal hearing. All participants acquired English prior to the age of 7 and completed a language background questionnaire on academic qualifications achieved in Welsh and English, length of language exposure, age at the time of exposure, ability to speak, write, and comprehend English and Welsh on a 10-point scale (with 10 being the highest level; see Table 1).

\section{Materials and design}

Stimuli were chosen from 28 semantic categories of the Van Overschelde et al. (2004) category norms. The 28 selected categories were arranged into pairs of yoked categories (e.g., animals-shapes or fruit-carpenter's tools). Only items from positions 13-24 in the category norm lists were used as TBR words, and only items from positions 1-12 were used as TBI words. List length was shorter than in Experiments 1 and 2 because of the need to use stimuli with appropriate Welsh-English translations. English TBI words and their Welsh translations were digitally recorded in a male voice.

Participant received 28 trials on which they were visually presented with 12 TBR words all drawn from one semantic category. On all trials, 12 auditory TBI words were interleaved with the TBR words. There were two experimental blocks of 14 trials each, 7 of which used semantically related TBI words and 7 of which used semantically unrelated TBI words. On the related trials, the auditory distractors were taken from the same category as the targets (e.g., animals). On the unrelated trials of the first block, the TBI items were taken from a yoked category (e.g., fruit category for TBR words and carpenter's tools category for TBI words). Similarly, on the unrelated trials of the second block, the TBR items were taken from categories used in the first block only as a source of TBI words (e.g., carpenter's tools), and TBI items were taken from their
Table 1 Language background questionnaire responses

Question

\begin{tabular}{ll}
\hline Have you taken GCSE Welsh? & $28 / 28$ Yes \\
Have you taken A Level Welsh? & $10 / 28$ Yes \\
Are your parents Welsh? & $19 / 28$ Yes \\
Which language do you speak at home? & $15 / 28$ Welsh \\
& $12 / 28$ English \\
& $1 / 28$ English and Welsh \\
& $M=42.9(S E=5.3)$ \\
What percentage of your & $M=2.61(S E=.42)$, \\
everyday speech is in Welsh? & range $=0-6$ \\
At which age did you first learn English? & $28 / 28$ Yes \\
& $11 / 28$ Yes \\
Have you taken GCSE English? & $M=57.1(S E=5.3)$ \\
Have you taken A Level English? & $M=9.1(S E=0.20)$ \\
What percentage of your everyday & $M=9.6(S E=0.15)$ \\
speech is in English? & $M=9.2(S E=0.19)$ \\
Rate your ability to speak Welsh & $M=8.8(S E=0.26)$ \\
Rate your ability to understand Welsh & $M=9.1(S E=0.21)$ \\
Rate your ability to read Welsh & $M=9.5(S E=0.17)$ \\
Rate your ability to write Welsh & $M=9.4(S E=0.18)$ \\
Rate your ability to speak English & $M=9.1(S E=0.22)$ \\
Rate your ability to understand English & $13 / 28$ Welsh \\
Rate your ability to read English & $14 / 28$ English \\
Rate your ability to write English & $1 / 28$ Both \\
Which is your strongest language? & \\
& \\
&
\end{tabular}

yoked categories (e.g., fruit). The related and unrelated conditions alternated within a block, and both the assignment of categories to conditions and whether a block initiated from related or unrelated condition were counterbalanced across participants.

Two blocks of 14 trials were used to introduce the manipulation of linguistic discriminability. In one block, participants were presented with TBI words in English, and in the other block, they were presented with TBI words in Welsh. The order of the blocks and the assignment of categories to a block were counterbalanced across participants. Visual TBR words were always presented in English, and perceptual discriminability was not manipulated in the present experiment; all conditions introduced modality difference between TBR and TBI words, which aimed at securing maximal perceptual discriminability. Thus, the experiment conformed to a 2 (semantic similarity of TBR and TBI words: related vs. unrelated) $\times 2$ (linguistic discriminability of TBR and TBI words: high vs. low) within-participants design.

\section{Procedure}

Participants were tested individually in soundproof booths, wearing headphones throughout. They were told to ignore 
any words they heard over the headphones and that they would not be asked about them at any point during the experiment. The TBR words were presented one at a time on the computer screen at the rate of $750 \mathrm{~ms}$ each, with a 750-ms interval during which TBI words were played over the headphones at $65 \mathrm{~dB}(\mathrm{~A})$. After all $12 \mathrm{TBR}$ words had been presented, the prompt "recall" appeared on the screen. Participants then had $30 \mathrm{~s}$ to write down on the response sheets as many target items as they could. Guessing was explicitly discouraged. After the 30 -s recall period, a tone in the headphones signaled the end of the trial. Pressing the space bar initiated presentation of the next list. One practice trial (in quiet) was given at the start of each block.

Results

\section{False recalls}

The means are presented in Fig. 5. A 2 (semantic similarity of TBR and TBI words: related vs. unrelated) $\times 2$ (linguistic discriminability of TBR and TBI words: high vs. low) repeated measures ANOVA yielded a significant main effect of semantic similarity, $F(1,27)=18.41, M S E=6.19$, partial $\eta^{2}=.41$, $p<.001$, but no effect of linguistic discriminability, $F(1,27)=$ $0.94, M S E=4.2$, partial $\eta^{2}=.03, p=.34$. There was no interaction, $F(1,27)=0.09, M S E=2.48$, partial $\eta^{2}=.003$, $p=.77$. Thus, against predictions, linguistic discriminability had no influence on false recalls, which remained more prevalent when TBI words were related to TBR words, as compared with when they were unrelated.

\section{Correct recalls}

The means are presented in Fig. 6. An ANOVA carried out on the items correctly recalled revealed a significant main effect of semantic similarity, $F(1,27)=38.17, M S E=30.49$, partial

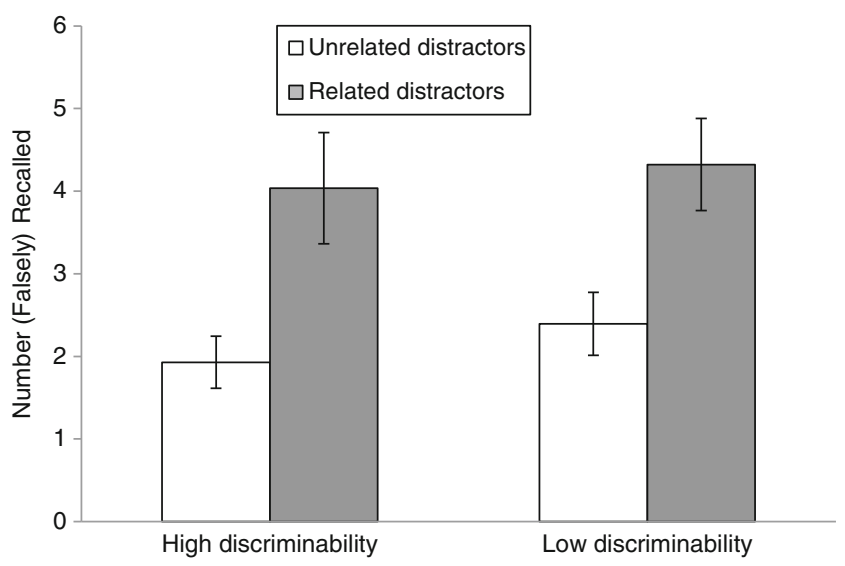

Fig. 5 The effects of semantic and linguistic relationships between TBR and TBI words on the number of intrusion errors (items falsely recalled) in Experiment 3. Error bars are standard errors

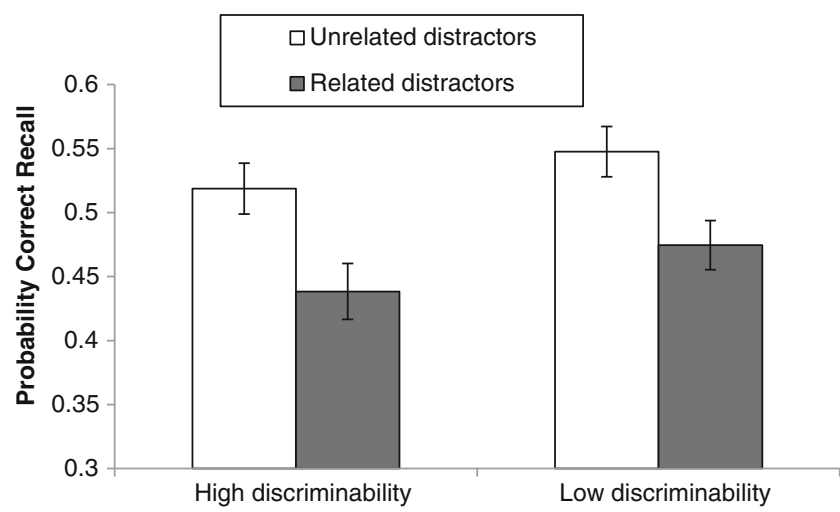

Fig. 6 The effects of semantic and linguistic relationships between TBR and TBI words on the probability of correct recall in Experiment 3. Error bars are standard errors

$\eta^{2}=.59, p<.001$, and also a significant main effect of linguistic discriminability, $F(1,27)=9.39, M S E=$ 22.25, partial $\eta^{2}=.26, p=.005$. There was no interaction, $F(1,27)=0.16, M S E=.002$, partial $\eta^{2}=.006$, $p=.69$. Thus, related TBI words once again reduced correct retrieval, as compared with unrelated TBI words. At the same time, high linguistic discriminability led to overall reduced performance, as compared with low linguistic discriminability.

\section{Discussion}

The present experiment aimed at testing whether the negative effects of semantic similarity of TBR and TBI words can be eliminated by enhancing nonsemantic discriminability of TBR and TBI words. The answer to this question is an unequivocal "no." Despite implementing study conditions that should result in the strongest possible perceptual discriminability of TBR and TBI items (by using different sensory modalities for these items) and additionally augmenting discriminability beyond a raw, sensory level with a linguistic feature in one of the conditions of our design (when TBR items were English and TBI items were Welsh), the memory performance was still uniformly worse when TBI words were semantically related to TBR words. This was reflected in both the elevated levels of erroneous recall and reduced levels of correct recall. These results testify to the crucial role of semantic factors in driving memory discrimination during retrieval and - in line with the results of the DRM paradigm (e.g., Schacter et al., 1999) - the present results indicate that errors arising from semantic relatedness of relevant and irrelevant information cannot be completely eliminated.

One unexpected finding was that high linguistic discriminability actually reduced memory performance, both when TBR and TBI words were semantically related and when they were unrelated, although this was observed for correct 
recall but not for erroneous recall. The reasons for this are unclear. It is possible that this linguistic effect is caused by some type of attentional reorientation during encoding. Some authors have argued that disruption in memory performance caused by auditory distractors is at least partly due to the fact that auditory distractors draw attention to themselves, leaving fewer attentional resources for encoding operations directed at TBR words (Bell, Buchner, \& Mund, 2008; Buchner, Rothermund, Wentura, \& Mehl, 2004). A study by Buchner and Erdfelder (2005) showed, in the context of a serial recall task, that disruption in memory is exacerbated when distractors are of lower frequency, presumably because low-frequency distractors are more effective in triggering attentional reorientation. It is possible that in the present study, TBI words presented in Welsh played the role of such low-frequency distractors. It is worth noting that the bilinguals tested in the present study rated, on average, the majority of their everyday speech to be English, and thus, they could be relatively less familiar with words in Welsh. Alternatively, there may have been attention-demanding (or otherwise disruptive) acoustic features of the Welsh language TBI stimuli that were absent from the English language TBI stimuli. The question of whether the generally more disruptive effects of Welsh speech, as compared with English speech, is due to attentional factors should be addressed by further studies, but it does not preclude us from drawing some specific conclusions about the nature of retrieval under conditions of semantic distraction.

\section{General discussion}

The present study investigated how semantic and nonsemantic features of relevant and irrelevant information stored in memory affect memory performance in the SD paradigm. The findings can be summarized as follows. First, all three experiments presented here demonstrated that when semantic discriminability is low (TBI words are semantically similar to TBR words), memory performance suffers, which is reflected in both the increased rate of intrusions and reduced correct retrieval. This occurs whether distractors are presented within modality (Experiments 1 and 2) or across modality (Experiment 3). Second, Experiments 1 and 2 demonstrated that the negative effects of low semantic discriminability for memory performance can be partially mitigated by increasing nonsemantic discriminability. In the face of high similarity of TBR and TBI words, introducing a perceptual feature that differentiated between TBR and TBI words led to better memory performance, manifested both by a reduction in erroneous retrieval and by an increase in correct retrieval. These effects occurred both when the SD paradigm was implemented in the visual (Experiment 1) and auditory
(Experiment 2) modalities. This directly demonstrates that perceptually differentiating - in this case, a series — of distractors from the TBR items along a single dimension impacts upon correct and erroneous recall. Third, the results of Experiment 3 testify to the potent role of semantic discriminability in memory retrieval by showing that even a very pronounced increase in nonsemantic discriminability, which included a modality difference between TBR and TBI words (TBR items were visual, TBI items were auditory) and also an additional linguistic dimension (TBR items were English words, TBI items were Welsh words) that differentiated between these words, does not eliminate the negative effects of reduced semantic discriminability. Together, the results show that reduced semantic discriminability of relevant and irrelevant information negatively affects memory and that nonsemantic discriminability can partially, but only partially, mitigate these negative effects. This failure to eliminate the SD effect completely by simultaneously providing modalitybased and lexically based cues for discrimination to offset categorical and contextual overlap between targets and distractors suggests that there may be limits to the usefulness of nonsemantic front-end control when items from within a single category are recalled in the presence of related distractors.

An important theoretical contribution of the present study lies in the simultaneous investigation of both erroneous and correct retrieval. Although these two facets of memory retrieval are commonly investigated in different types of procedures, the present study demonstrates how erroneous and correct retrieval can be investigated within a single methodology. Previous studies of source-directed retrieval (Alban \& Kelley, 2012; Kantner \& Lindsay, 2013) found only weak evidence for front-end control, perhaps because these studies utilized recognition memory wherein re-presentation of the items may have counteracted the effects of front-end control by automatically reinstating those items within memory. Here, we employed a recall procedure and were able to show discriminability effects on correct recall (Experiments 1 and 2), which are predicted only by front-end, not back-end, control mechanisms. Similarly, in the DRM paradigm, even when recall is employed, the critical lure is only a single item, so that any effect it may have on correct recall of TBR items must be limited, since consideration of the critical lure at retrieval increases the nominal memory load only by a single item, which is unlikely to show much effect on recall of already long (considerably super-span) lists. The SD paradigm, in contrast, introduces multiple distractors to the participants, and therefore, the effects on correct recall are much more marked. The implementation of the memory-as-discrimination framework with front-end control straightforwardly predicts that changes in one of the aforementioned aspects of retrieval should be paralleled by changes in the other aspect. Two experiments demonstrated exactly such a pattern of simultaneous changes 
in erroneous and correct retrieval resulting from manipulation of discriminability of relevant and irrelevant information. This is not to say that erroneous and correct retrieval are invariably linked in all circumstances. There is an abundance of studies demonstrating factors uniquely affecting only one aspect of retrieval. Nonetheless, it remains the case that correct and erroneous retrieval are commonly linked (for similar observations, see Spillers \& Unsworth, 2011; Unsworth, Spillers, \& Brewer, 2012), and thus the assumption of front-end control system for memory discrimination has much to offer for investigation of memory processes under conditions of distraction.

Given that the SD paradigm was originally conceived as an extension of investigations of the effects of irrelevant sound on immediate recall (Neely \& LeCompte, 1999), it is legitimate to inquire to what extent the SD effects that were successfully moderated here were the consequence of memorial processes per se and to what extent they can be considered attentional in origin. For example, it has been suggested that augmenting stimuli with additional perceptual cues may counteract age-related decline in the ability to inhibit irrelevant information (Hasher \& Zacks, 1988). We view this possibility as unlikely, however, because - although there is prima facie evidence that semantic distractors may attract inhibition in our paradigm (Marsh et al., 2012; Marsh et al., in press) - it is far from established that inhibition of the distractors is responsible for the SD effect. A study by Hanczakowski et al. (2013) failed to find an SD effect in a postrecall recognition memory test, which would be expected if the presence of the distractors either attracted attention during encoding or was a consequence of competitive retrieval at recall. Contrariwise, the $\mathrm{SD}$ effect is present even when distractors are presented subsequent to the presentation of the target items (Marsh et al., 2008), which would not be expected if it were a consequence of divided attention at study.

It is also worth noting that we are examining specifically semantic distraction, which sets up the requirement to discriminate between semantically and categorically related items independently of any nonsemantic distraction effects that may be observed for other reasons. This raises the further possibility that the SD paradigm might be viewed as more akin to the buildup of PI over time from categorically related stimuli. Our distractors are presented in the same episode as to-be-remembered items, and so the task is really not about distinguishing between information from the current episode or trial and previous episodes or trials (as in investigations of PI) but about distinguishing between relevant and irrelevant information within the same episode/trial. Whereas we would normally expect this to be achieved by means of categorical and contextual cues, it turns out (Experiments 1 and 2) that this process can also be augmented by perceptual cues operating as part of a front-end control mechanism. It is also of interest that a language-shift manipulation sufficient to produce release from PI in a previous study (Goggin \& Wickens, 1971) failed to eliminate the SD effect (Experiment 3), suggesting that perhaps the front-end mechanism whereby perceptual cues that distinguish relevant TBR items from irrelevant, but semantically related, TBI items on a single trial might differ in some respects from those used to release the buildup of PI by means of a category shift.

In a related series of studies, Tehan and colleagues (Tehan \& Humphreys, 1996; Tehan, Humphreys, Tolan, \& Pitcher, 2004; Tolan \& Tehan, 1999, 2002) have also used semantic and other cues to manipulate PI. In these cases, Tehan and colleagues added more cues to help avoid errors or to cause errors, distributing the codes among several words to exclude the possibility of any strategic effect. Interestingly, in one of these papers (Tolan \& Tehan, 2002), irrelevant speech was used to modify semantic cuing and regulate PI. In this study, however, interference consisted of retrieval of an item from the wrong list, and there were no effects on correct retrieval. Provision of the phonemic codes thus appears to have changed some of the omission errors into intrusion errors without affecting correct recalls. This result speaks more to the changing nature of error when the possibility of strategic control is minimized, rather than the strategic reduction in errors of both types (false recall and failure to recall) as examined here.

To conclude, the present study joins the growing body of theoretical and empirical works showing that memory retrieval can be understood as a process of discriminating between relevant and irrelevant information at the point at which retrieval is initiated. The irrelevant information that is highly disruptive for this process is information related to relevant information by its semantic content. When one is trying to recall where one's car is parked, the event of parking on this day has to be discriminated from all other semantically related instances of parking a car that intrude into memory and make it difficult to recall necessary information. However, the process of remembering can, to a certain extent, be aided by details that differentiate between semantically similar events. To discriminate effectively is not just a matter of perceptually enriching the TBR information; the TBR information must actively be differentiated from the TBI item, as here. It could help, for example, to start remembering with today's weather and, thus, exclude all instances of parking that took place under different weather conditions, thus increasing the chances that necessary information will be eventually retrieved.

Author Note C. Philip Beaman, School of Psychology and Clinical Language Sciences, University of Reading, UK.

Maciej Hanczakowski, Helen M. Hodgetts \& Dylan M. Jones, School of Psychology, Cardiff University, UK. Dylan Jones is also adjunct Professor at the Department of Psychology at the University of Western Australia.

John E. Marsh, School of Psychology, University of Central Lancashire, Preston, UK. 
Order of authorship for the first four authors was determined alphabetically, and no precedence or inequality of contribution is implied or should be inferred. This research was carried out while John E. Marsh was employed by the School of Psychology, Cardiff University, and was supported by ERSC grant RES-062-23-1752 awarded to Dylan Jones and Philip Beaman. We thank Rob Hughes for recording the Welsh language distractors for Experiment 3. Correspondence can be addressed to Philip Beaman at the School of Psychology \& Clinical Language Sciences, University of Reading, Earley Gate, Whiteknights, Reading, RG6 6AL, United Kingdom. Email may be sent to c.p.beaman@reading.ac.uk.

\section{References}

Alban, M. W., \& Kelley, C. M. (2012). Variations in constrained retrieval. Memory and Cognition, 40, 681-692.

Beaman, C. P. (2004). The irrelevant sound phenomenon revisited: What role for working memory capacity? Journal of Experimental Psychology: Learning, Memory, and Cognition, 30, 1106-1118.

Bell, R., Buchner, A., \& Mund, I. (2008). Age-related differences in irrelevant-speech effects. Psychology and Aging, 23, 377-391.

Brainerd, C. J., Reyna, V. F., Wright, R., \& Mojardin, A. H. (2003). Recollection rejection: False-memory editing in children and adults. Psychological Review, 110, 762-784.

Brainerd, C. J., Wright, R., Reyna, V. F., \& Mojardin, A. H. (2001). Conjoint recognition and phantom recollection. Journal of Experimental Psychology: Learning, Memory, and Cognition, 27, 307-327.

Buchner, A., \& Erdfelder, E. (2005). Word frequency of irrelevant speech distracters affects serial recall. Memory and Cognition, $33,86-97$.

Buchner, A., Rothermund, K., Wentura, D., \& Mehl, B. (2004). Valence of distractor words increases the effects of irrelevant speech on serial recall. Memory and Cognition, 32, 722731.

Budson, A. E., Droller, D. B. J., Dodson, C. S., Schacter, D. L., Rugg, M. D., Holcomb, P. J., \& Daffner, K. R. (2005). Electrophysiological dissociation of picture versus word encoding: The distinctiveness heuristic as a retrieval orientation. Journal of Cognitive Neuroscience, 17, 1181-1193.

Deese, J. (1959). On the prediction of occurrence of particular verbal intrusions in immediate recall. Journal of Experimental Psychology, $58,17-22$.

Dodson, C. S., \& Schacter, D. L. (2001). "If I had said it I would have remembered it": Reducing false memories with a distinctiveness heuristic. Psychonomic Bulletin and Review, 8, 155-161.

Dodson, C. S., \& Schacter, D. L. (2002). When false recognition meets metacognition: The distinctiveness heuristic. Journal of Memory and Language, 46, 782-803.

Gallo, D. A., Weiss, J. A., \& Schacter, D. L. (2004). Reducing false recognition with criteria recollection tests: Distinctiveness heuristic versus criterion shifts. Journal of Memory and Language, 51, 473-493.

Goggin, J., \& Wickens, D. D. (1971). Proactive interference and language change in short-term memory. Journal of Verbal Learning and Verbal Behavior, 10, 453-458.

Goh, W. D., \& Lu, S. H. X. (2012). Testing the myth of the encodingretrieval match. Memory and Cognition, 40, 28-39.

Goldsmith, M., \& Koriat, A. (2008). The strategic regulation of memory accuracy and informativeness. In A. Benjamin \& B. Ross (Eds.), Psychology of learning and motivation, Vol. 48: Memory use as skilled cognition (pp. 1-60). San Diego, CA: Elsevier.
Halamish, V., Goldsmith, M., \& Jacoby, L. L. (2012). Sourceconstrained recall: Front-end and back-end control of retrieval quality. Journal of Experimental Psychology: Learning, Memory, and Cognition, 38, 1-15.

Hanczakowski, M., Beaman, C. P., \& Jones, D. M. (submitted). On the mechanisms of the semantic similarity effect in auditory distraction.

Hanczakowski, M., \& Mazzoni, G. (2011). Both differences in encoding processes and monitoring at retrieval reduce false alarms when distinctive information is studied. Memory, 19, 280-289.

Hanley, J. R., \& Bakopoulou, E. (2003). Irrelevant speech, articulatory suppression, and phonological similarity: A test of the phonological loop model and the feature model. Psychonomic Bulletin and Review, 10, 435-444.

Hasher, L., \& Zacks, R. T. (1988). Working memory, comprehension and aging: A review and a new view. In G. H. Bower (Ed.), The psychology of learning and motivation (Vol. 22). San Diego: Academic Press.

Higham, P. A., \& Tam, H. (2005). Generation failure: Estimating metacognition in cued recall. Journal of Memory and Language, 52, 595-617.

Hunt, R. R. (2003). Two contributions of distinctive processing to accurate memory. Journal of Memory and Language, 48, 811825.

Israel, L., \& Schacter, D. L. (1997). Pictorial encoding reduces false recognition of semantic associates. Psychonomic Bulletin and Review, 4, 577-581.

Jacoby, L. L., Kelley, C. M., \& Dywan, J. (1989). Memory attributions. In H. L. Roediger \& F. I. M. Craik (Eds.), Varieties of memory and consciousness: Essays in honour of Endel Tulving (pp. 391-422). Hillsdale, NJ: Erlbaum.

Jacoby, L. L., Kelley, C. M., \& McElree, B. (1999). The role of cognitive control: Early selection versus late correction. In S. Chaiken \& Y. Trope (Eds.), Dual process theories in social psychology (pp. 383-400). New York: Guilford Press.

Jacoby, L. L., Shimizu, Y., Daniels, K. A., \& Rhodes, M. G. (2005a). Modes of cognitive control in recognition and source memory: Depth of retrieval. Psychonomic Bulletin and Review, 12, 852-857.

Jacoby, L. L., Shimizu, Y., Velanova, K., \& Rhodes, M. G. (2005b). Age differences in depth of retrieval: Memory for foils. Journal of Memory and Language, 52, 493-504.

Kantner, J., \& Lindsay, D. S. (2013). Top-down constraint on recognition memory. Memory \& Cognition, 41, 465-479.

Koriat, A., \& Goldsmith, M. (1996). Monitoring and control processes in the strategic regulation of memory accuracy. Psychological Review, 103, 490-517.

Leboe, J. P., \& Whittlesea, B. W. A. (2002). The inferential basis of familiarity and recall: Evidence for a common underlying process. Journal of Memory and Language, 46, 804-829.

Marsh, J. E., Beaman, C. P., Hughes, R. W., \& Jones, D. M. (2012). Inhibitory control in memory: Evidence for negative priming in free recall. Journal of Experimental Psychology: Learning, Memory, and Cognition, 38, 1377-1388.

Marsh, J. E., Hughes, R. W., \& Jones, D. M. (2008). Auditory distraction in semantic memory: A process-based approach. Journal of Memory and Language, 58, 682-700.

Marsh, J. E., Hughes, R. W., \& Jones, D. M. (2009). Interference by process, not content, determines semantic auditory distraction. Cognition, 110, 23-38.

Marsh, J. E., Sörqvist, P., Beaman, C. P., \& Jones, D. M. (in press) Auditory distraction eliminates retrieval induced forgetting: Implications for the processing of unattended sound. Experimental Psychology. doi:10.1027/1618-3169/a000210

McCabe, D. P., \& Smith, A. D. (2006). The distinctiveness heuristic in false recognition and false recall. Memory, 14, 570-583. 
Nairne, J. S. (1990). A feature model of immediate memory. Memory and Cognition, 18, 251-269.

Nairne, J. S. (2002). The myth of the encoding-retrieval match. Memory, 10, 389-395.

Nairne, J. S. (2005). The functionalist agenda in memory research. In A. F. Healy (Ed.), Experimental cognitive psychology and its applications (pp. 115-126). Washington, DC: American Psychological Association.

Neely, C. B., \& LeCompte, D. C. (1999). The importance of semantic similarity to the irrelevant speech effect. Memory and Cognition, 27, 37-44.

Poirier, M., Nairne, J. S., Morin, C., Zimmermann, F. G. S., Koutmeridou, K., \& Fowler, J. (2012). Memory as discrimination: A challenge to the encoding-retrieval match principle. Journal of Experimental Psychology: Learning, Memory, and Cognition, 38, 16-29.

Roediger, H. L., \& McDermott, K. B. (1995). Creating false memories: Remembering words not presented in lists. Journal of Experimental Psychology: Learning, Memory, and Cognition, 21, 803-814.

Salamé, P., \& Baddeley, A. D. (1982). Disruption of short-term memory by irrelevant speech: Implications for the structure of working memory. Journal of Verbal Learning and Verbal Behavior, 21, $150-164$.

Schacter, D. L., Israel, L., \& Racine, C. (1999). Suppressing false recognition in younger and older adults: The distinctiveness heuristic. Journal of Memory and Language, 40, 1-24.

Shimizu, Y., \& Jacoby, L. L. (2005). Similarity-guided depth of retrieval: Constraining at the front-end. Canadian Journal of Experimental Psychology, 59, 17-21.

Spillers, G. J., \& Unsworth, N. (2011). Are the costs of directed forgetting due to failures of sampling or recovery? Exploring the dynamics of recall in list-method directed forgetting. Memory and Cognition, 39, 403-411.

Tehan, G., \& Humphreys, M. S. (1996). Cuing effects in short-term recall. Memory and Cognition, 24, 719-732.

Tehan, G., Humphreys, M. S., Tolan, G. A., \& Pitcher, C. (2004). The role of context in producing item interactions and false memories. Journal of Experimental Psychology: Learning, Memory, and Cognition, 30, 107-119.

Tolan, G. A., \& Tehan, G. (1999). Determinants of short-term forgetting: decay, retroactive interference or proactive interference? International Journal of Psychology, 34, 285-292.

Tolan, G. A., \& Tehan, G. (2002). Testing feature interaction: betweenstream irrelevant speech effects in immediate recall. Journal of Memory and Language, 46, 562-585.

Unsworth, N., Spillers, G. J., \& Brewer, G. A. (2012). Evidence for noisy contextual search: Examining the dynamics of list-beforelast recall. Memory, 20, 1-13.

Van Overschelde, J. P., Rawson, K. A., \& Dunlosky, J. (2004). Category norms: An updated and expanded version of the Battig and Montague (1969) norms. Journal of Memory and Language, 50, 289-335.

Watkins, O. C., \& Watkins, M. J. (1975). Build-up of proactive inhibition as a cue overload effect. Journal of Experimental Psychology: Human Learning \& Memory, 104, 442-452.

Whittlesea, B. W. A. (1997). Production, evaluation and preservation of experiences: Constructive processing in remembering and performance tasks. In D. Medin (Ed.), The psychology of learning and motivation ((pp, Vol. 37, pp. 221-264). New York: Academic Press.

Wickens, D. D., Born, D. G., \& Allen, C. K. (1963). Proactive inhibition and item similarity in short-term memory. Journal of Verbal Learning and Verbal Behavior, 2, 440-445. 